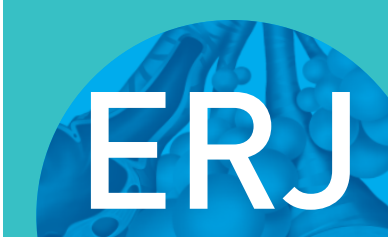

open research

\title{
Constrictive bronchiolitis in diffuse idiopathic pulmonary neuroendocrine cell hyperplasia
}

\author{
Bilal F. Samhouri ${ }^{1}$, Natalya Azadeh², Thorvardur R. Halfdanarson ${ }^{3}$, Eunhee S. Yi $\mathbb{\circledR}^{4}$ \\ and Jay H. Ryu ${ }^{1}$
}

Affiliations: 'Dept of Pulmonary and Critical Care Medicine, Mayo Clinic, Rochester, MN, USA. ${ }^{2}$ Dept of Pulmonary and Critical Care Medicine, Mayo Clinic, Phoenix, AZ, USA. ${ }^{3}$ Division of Medical Oncology, Mayo Clinic, Rochester, MN, USA. ${ }^{4}$ Depts of Laboratory Medicine and Pathology, Mayo Clinic, Rochester, MN, USA.

Correspondence: Jay H. Ryu, Division of Pulmonary and Critical Care Medicine, Gonda 18 South, Mayo Clinic, 200 First St SW, Rochester, MN 55905, USA. E-mail: ryu.jayवmayo.edu.

\section{ABSTRACT}

Background: Diffuse idiopathic pulmonary neuroendocrine cell hyperplasia (DIPNECH) is characterised by multifocal proliferation of neuroendocrine cells and belongs in the spectrum of pulmonary neuroendocrine tumours. Some patients with DIPNECH develop airflow obstruction but the relationship between the two entities remains unclear.

Methods: We performed a computer-assisted search of the Mayo Clinic's electronic medical records for biopsy-proven cases of DIPNECH. We extracted clinical, pulmonary function, imaging and histopathological data along with treatments and outcomes.

Results: Among 44 patients with DIPNECH 91\% were female and the median age was 65 years (interquartile range 56-69 years); 73\% were never-smokers. Overall, 38 patients ( $86 \%$ ) had respiratory symptoms including cough (68\%) and dyspnoea (30\%); 45\% were previously diagnosed to have asthma or COPD. Pulmonary function testing showed an obstructive pattern in 52\%, restrictive pattern in $11 \%$, mixed pattern in $9 \%$, nonspecific pattern in $23 \%$, and was normal in $5 \%$. On chest computed tomography scan, $95 \%$ manifested diffuse nodules and $77 \%$ manifested mosaic attenuation. For management, $25 \%$ of patients were observed without pharmacological therapy, 55\% received an inhaled bronchodilator, $41 \%$ received an inhaled corticosteroid, 32\% received octreotide; systemic steroids, azithromycin, or combination chemotherapy was employed in four patients (9\%). Of 24 patients with available follow-up pulmonary function tests, 50\% remained stable, 33\% worsened and $17 \%$ improved over a median interval of 21.3 months (interquartile range 9.7-46.9 months).

Conclusion: DIPNECH occurs mostly in women and manifests diffuse pulmonary nodules and mosaic attenuation on imaging. It is commonly associated with airflow obstruction due to constrictive bronchiolitis, which manifests limited response to current pharmacological therapy.

@ERSpublications

Diffuse idiopathic pulmonary neuroendocrine cell hyperplasia (DIPNECH) is an underrecognised cause of obstructive lung disease in women. Constrictive bronchiolitis associated with DIPNECH manifests limited response to currently employed therapies. https://bit.ly/3c3RZoe

Cite this article as: Samhouri BF, Azadeh N, Halfdanarson TR, et al. Constrictive bronchiolitis in diffuse idiopathic pulmonary neuroendocrine cell hyperplasia. ERJ Open Res 2020; 6: 00527-2020 [https://doi.org/10.1183/23120541.00527-2020].

This article has supplementary material available from openres.ersjournals.com.

Received: 26 July 2020 | Accepted after revision: 9 Sept 2020

Copyright $\odot$ ERS 2020. This article is open access and distributed under the terms of the Creative Commons Attribution Non-Commercial Licence 4.0. 


\section{Introduction}

Pulmonary neuroendocrine cells (PNECs) are dispersed throughout the lungs and constitute $<1 \%$ of the cells in adult human lungs [1]. The majority of PNECs are located in the bronchi, whereas others are situated in terminal bronchioles and alveolar ducts [2]. The function of PNECs is not entirely clear but studies have suggested that they play a role in detecting hypoxaemia [3], immunomodulation [4] and regeneration of lung epithelial cells [5]. Hyperplasia of PNECs can occur secondary to various forms of injuries (e.g. chronic hypoxia associated with living at high altitudes and COPD) or can be idiopathic $[6,7]$.

In 1992, Aguayo et al. [8] described six patients exhibiting multifocal hyperplasia of PNECs with associated peribronchiolar fibrosis on histological examination combined with clinical and radiological features suggestive of small airway disease. This disease entity was referred to as "idiopathic diffuse hyperplasia of pulmonary neuroendocrine cells and airways disease". In the 2001 World Health Organization (WHO) classification of lung tumours, this entity was termed "diffuse idiopathic pulmonary neuroendocrine cell hyperplasia" (DIPNECH) [9].

Additional studies followed describing the association of DIPNECH with obstructive small airways disease (constrictive bronchiolitis (CB)) through mechanisms that will be discussed later in this paper [8, 10, 11]. Thus, patients with DIPNECH may manifest respiratory symptoms, an obstructive pattern on pulmonary function testing (PFT) and mosaic pattern (i.e. "patchwork regions of differing attenuation") [12] with patchy air trapping on chest computed tomography (CT) [10, 13]. The term "DIPNECH syndrome" has been proposed for the combination of these features occurring with histological DIPNECH [14].

Because of the rarity of this recently described entity, we aimed to further characterise the relationship between small airways disease (CB) and DIPNECH including clinical, physiological and radiological correlates.

\section{Methods}

We conducted a search of Mayo Clinic electronic medical records for cases of biopsy-proven DIPNECH seen at the three Mayo Clinic campuses in Minnesota, Florida and Arizona in the United States. We manually extracted demographic, clinical, laboratory, pulmonary function and imaging data from electronic medical records, including review of radiological studies. The pathological diagnosis of DIPNECH was confirmed by the finding on lung biopsy of multifocal proliferation of PNECs involving the bronchial or bronchiolar epithelium (figure 1) [15].
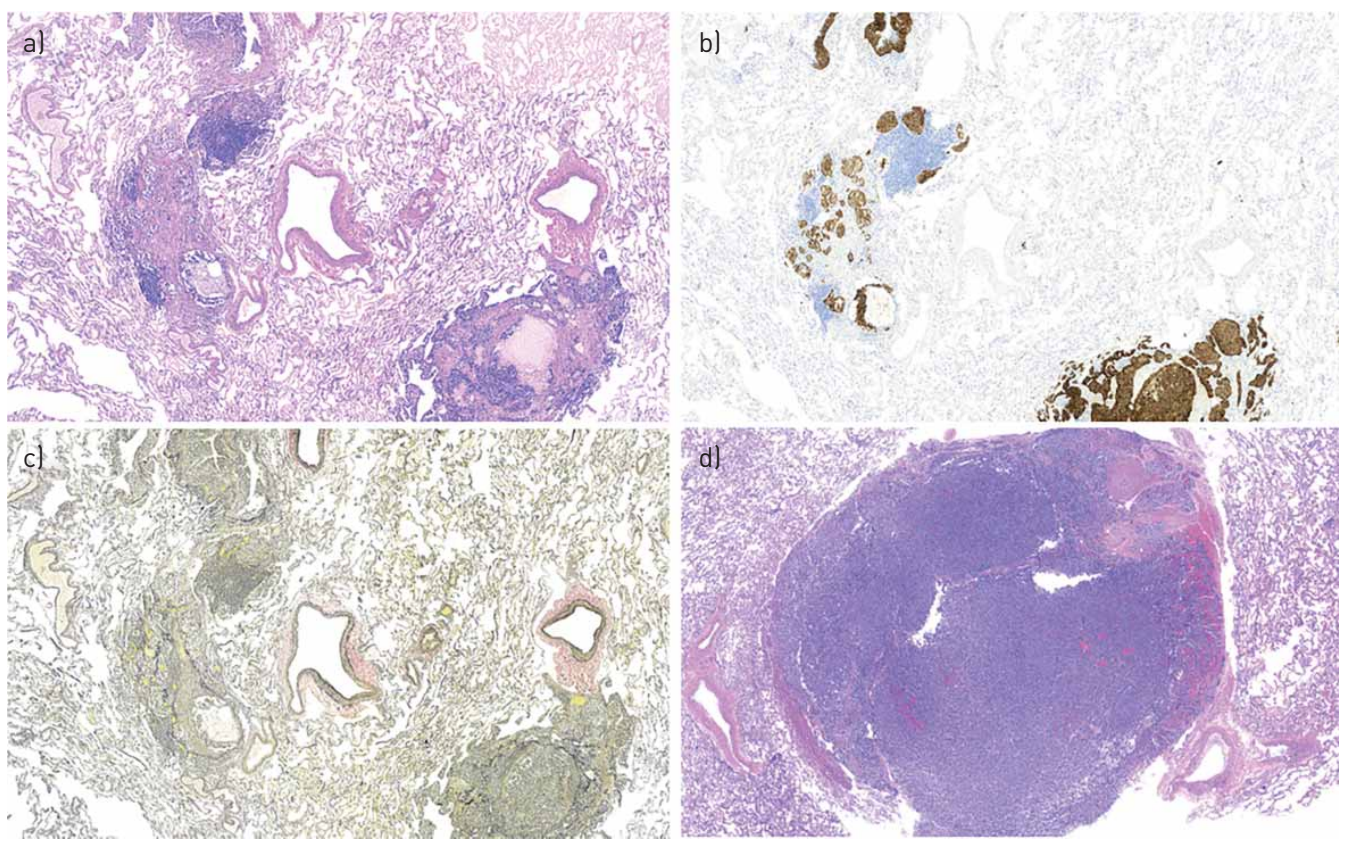

FIGURE 1 a) Normal-appearing alveolar parenchyma with multiple small airways involved by neuroendocrine cell hyperplasia (haematoxylin and eosin, $\times 40$ original magnification). b) Proliferating neuroendocrine cells highlighted by chromogranin immunostaining (anti-chromogranin antibody, $\times 40$ ). c) Partial or complete obliteration of the bronchiolar lumens by proliferating neuroendocrine cells and mucosal fibrosis (Verhoeff-Van Gieson, $\times 40$ ). d) Carcinoid tumour arising in a background of diffuse neuroendocrine cell hyperplasia (haematoxylin and eosin, $\times 20$ ). 
PFT results were classified as normal, obstructive, restrictive, mixed or nonspecific patterns. An obstructive pattern was defined as forced expiratory volume in $1 \mathrm{~s}\left(\mathrm{FEV}_{1}\right) /$ forced vital capacity $(\mathrm{FVC})<$ lower limit of normal (LLN) [16]. The restrictive pattern was defined by total lung capacity (TLC) $<$ LLN [16]. The mixed pattern (obstructive and restrictive) was diagnosed when $\mathrm{FEV}_{1} / \mathrm{FVC}<\mathrm{LLN}$ and TLC $<$ LLN [16]. The nonspecific pattern was defined as $\mathrm{FEV}_{1}$ and/or FVC $<\mathrm{LLN}$, with a normal $\mathrm{FEV}_{1} / \mathrm{FVC}$ ratio and a TLC value that was $>$ LLN or not obtained [17]. Positive bronchodilator response was defined as an increase of $>12 \%$ and $>200 \mathrm{~mL}$ in $\mathrm{FEV}_{1}$ and/or FVC following the inhalation of a $\beta_{2}$-agonist [16]. Air trapping was defined as residual volume (RV) $>120 \%$ predicted. Per the American Thoracic Society/European Respiratory Society guidelines, the severity of obstructive, restrictive and mixed PFT patterns was graded based on the $\mathrm{FEV}_{1}$, expressed as a percentage of the predicted value: 1) mild: $\mathrm{FEV}_{1} \geqslant 70 \%$; 2) moderate: $\mathrm{FEV}_{1}$ 60-69\%; 3) moderately severe: $\mathrm{FEV}_{1}$ 50-59\%; 4) severe: FEV1 35-49\%; and 5) very severe: $\mathrm{FEV}_{1}<35 \%[16,18]$. Reduced diffusing capacity of carbon monoxide $\left(D_{\mathrm{LCO}}\right)$ was defined as $D_{\mathrm{LCO}}<\mathrm{LLN}$. A significant change on follow-up PFT was defined as a change of $\geqslant 15 \%$ for $\mathrm{FEV}_{1}$ and/or FVC, and $\geqslant 10 \%$ for $D_{\mathrm{LCO}}[16]$.

Counts and percentages were used for descriptive statistics. Median and interquartile range (IQR) 25-75 were used to describe central tendency measures. A Chi-squared test was used for analysing correlations between categorical variables. A $\mathrm{p}<0.05$ was used for determination of statistical significance.

This study was approved by Mayo Clinic’s Institutional Review Board (no. 17-000885).

\section{Results}

We identified 44 patients with biopsy-proven DIPNECH; 91\% were women and 73\% were never-smokers (table 1). Most patients (89\%) were white. Median age at diagnosis was 65 years (IQR 56-69). Past medical history included cancer in six patients (14\%), skin cancer in three, breast cancer in one, lung adenocarcinoma in one and a combination of papillary thyroid cancer, retroperitoneal sarcoma and melanoma in one.

Most patients (86\%) presented for evaluation of respiratory symptoms, most commonly cough (table 1). Symptoms were present for a median duration of 120 months (IQR 24-240) prior to diagnosis. Six patients (14\%) had no respiratory symptoms but presented for evaluation of pulmonary nodules incidentally identified on a chest CT scan performed for another indication. On lung auscultation, wheezes or crackles were described in $14 \%$, distant breath sounds or prolonged expiratory phase in $11 \%$, but normal breath sounds in the remaining $75 \%$. The major metabolite of serotonin, 5-hydroxyindoleacetic acid (5-HIAA), was measured in six patients and was elevated in one.

Chest CT scans were available for all patients (table 2). Nearly all patients (95\%) manifested numerous small bilateral pulmonary nodules (figure 2a), while two patients did not; one patient manifested subtle regions of ground-glass attenuation and one patient manifested an enlarging single nodule $(1.7 \mathrm{~cm}$ in size) that was found to represent a carcinoid tumour, with the resected specimen revealing a background of DIPNECH and CB. Mosaic attenuation with air trapping (figure $2 \mathrm{~b}$ ) was present in $77 \%$ of patients, all of whom also manifested diffuse pulmonary nodules.

\section{TABLE 1 Demographic and clinical characteristics $(n=44)$}

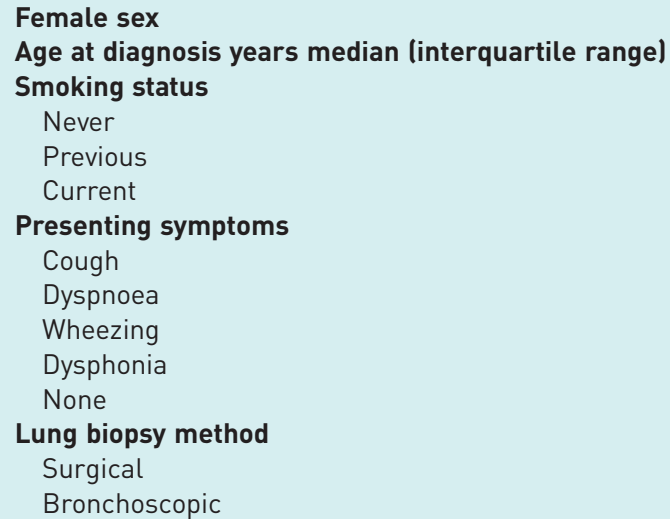




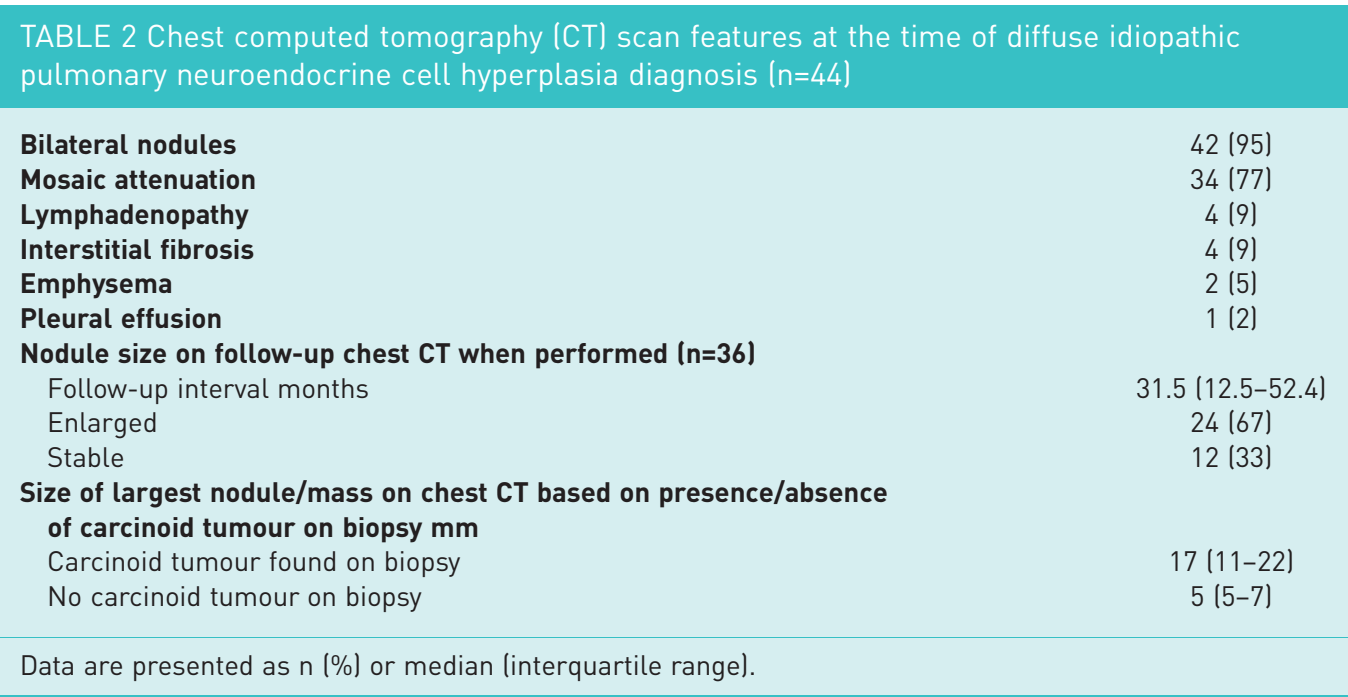

One or more PFT results were available for all patients and demonstrated an obstructive pattern in 23 patients $(52 \%)$, restrictive pattern in $5(11 \%)$, mixed pattern in $4(9 \%)$, nonspecific pattern in $10(23 \%)$ and normal in $2(5 \%)$. The PFT data are illustrated in table 3 and supplementary table 1 . As the focus of this study is the characterisation of the small airway disease associated with DIPNECH, we divided patients into two groups based on the presence (isolated obstructive or mixed obstructive-restrictive pattern) or absence of obstructive ventilatory defect on PFT. Approximately one-half (52\%) of 27 patients with an obstructive component had severe to very severe impairment. The $D_{\mathrm{LCO}}$ value was available for all patients and was reduced in $48 \%$ of patients.

Patients with obstruction manifested mosaic attenuation on chest CT scan with a higher frequency than patients without obstruction ( $82 \%$ versus $65 \%$, respectively, $\mathrm{p}=0.21)$, but manifested $\mathrm{CB}$ on biopsy with a comparable frequency to patients without obstruction ( $44 \%$ versus $53 \%$, respectively, $\mathrm{p}=0.58$ ) (table 4 and supplementary table 2).

Diagnostic lung specimens were obtained surgically in $95 \%$ of patients, while $5 \%$ were obtained through transbronchial biopsy. Histopathological findings are summarised in table 4. Aside from DIPNECH, carcinoid "tumourlets" (focal collection of PNECs $<5 \mathrm{~mm}$ in size) [15] were identified in 34 patients (77\%) and carcinoid tumours in $16(36 \%)$.

Among the 16 patients who underwent carcinoid tumour resection, 5 patients (31\%) reported symptomatic improvement following surgery, 9 (56\%) reported no change and 2 (13\%) had no respiratory symptoms before or after surgery. Overall, 10 patients had a follow-up PFT; 5 (50\%) declined, 2 (20\%) improved and $3(30 \%)$ remained stable.
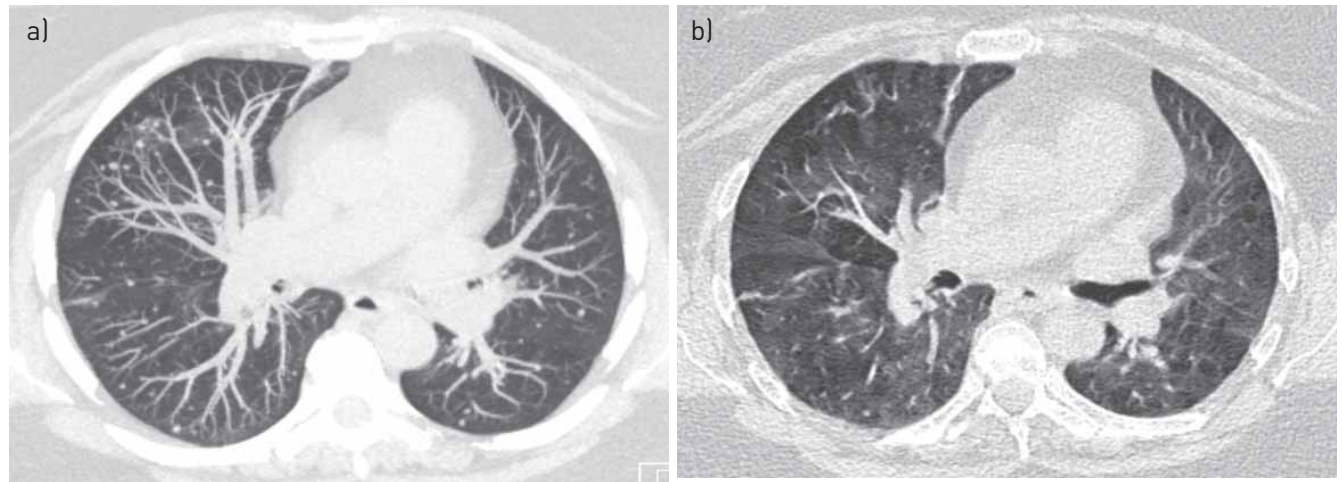

FIGURE 2 Chest computed tomography images of a 60-year-old female patient with diffuse idiopathic pulmonary neuroendocrine cell hyperplasia. al Maximum intensity projection image demonstrating multiple bilateral micronodules. b) Mosaic attenuation. 


\begin{tabular}{|c|c|c|c|}
\hline & Obstruction & No obstruction & Overall \\
\hline Patients & $27(61)$ & 17 (39) & 44 \\
\hline \multicolumn{4}{|l|}{ Severity of disease graded by $\mathrm{FEV}_{1}(\mathrm{n}=44)$} \\
\hline$\geqslant 70 \%$ predicted & $3(11)$ & $5(29)$ & $8(18)$ \\
\hline $60-69 \%$ predicted & $2(7)$ & 5 (29) & $7(16)$ \\
\hline $50-59 \%$ predicted & $8(30)$ & $3(18)$ & $11(25)$ \\
\hline $35-49 \%$ predicted & $7(26)$ & $4(24)$ & $11(25)$ \\
\hline$<35 \%$ predicted & $7(26)$ & $0(0)$ & $7(16)$ \\
\hline \multicolumn{4}{|l|}{ Bronchodilator responsiveness when tested $(n=37)$} \\
\hline Present & $12(52)$ & $1(7)$ & $13(35)$ \\
\hline Absent & $11(48)$ & $13(93)$ & $24(65)$ \\
\hline \multicolumn{4}{|c|}{ Air trapping among patients with RV measurement ( $n=43$ ) } \\
\hline Present & $22(85)$ & $2(12)$ & $24(56)$ \\
\hline Absent & $4(15)$ & $15(88)$ & $19(44)$ \\
\hline \multicolumn{4}{|l|}{$D_{\text {LCo }}(n=44)$} \\
\hline Reduced $D_{\mathrm{Lco}}$ & $13(48)$ & 8 (47) & $21(48)$ \\
\hline$D_{\text {LCo }} \%$ predicted median (interquartile range) & $77(66-86)$ & 77 (65-99) & $76(65-92)$ \\
\hline
\end{tabular}

Data are presented as $\mathrm{n}(\%)$ unless otherwise stated. $\mathrm{FEV}_{1}$ : forced expiratory volume in $1 \mathrm{~s}$; RV: residual volume; $D_{\text {LCO }}$ : diffusing capacity of carbon monoxide.

Monitoring (clinical, radiological and with serial PFTs) without pharmacological therapy was recommended for 11 patients (25\%), whereas pharmacotherapy was recommended for 33 (75\%) (supplementary table 3 ). An inhaled bronchodilator $\left(\beta_{2}\right.$-agonist and/or an antimuscarinic agent) was prescribed in 24 patients (55\%); 18 (75\%) of whom manifested an obstructive component on PFT. Of all patients prescribed a bronchodilator, only four (17\%) reported symptomatic improvement, two of whom manifested a positive bronchodilator response on PFT. It is worth noting that a positive bronchodilator response on PFT was not consistently associated with symptomatic response to inhaled $\beta_{2}$-agonists; seven patients with a positive bronchodilator response received a $\beta_{2}$-agonist without improvement.

An inhaled corticosteroid (ICS) was prescribed in 18 patients (41\%); 4 (22\%) experienced subjective improvement and 3 (25\%) of 12 patients with available follow-up PFT data demonstrated improvement on PFT. One patient received a systemic steroid (prednisone) and reported stable symptoms.

TABLE 4 Chest computed tomography (CT) scan and pathology findings for patients with and without obstruction on pulmonary function testing

\begin{tabular}{lccc} 
& Obstruction & No obstruction & Overall \\
\hline $\begin{array}{l}\text { Patients } \\
\text { Chest CT scan findings }\end{array}$ & $27(61)$ & $17(39)$ & $44(100)$ \\
$\quad$ Diffuse nodules & $26(96)$ & $16(94)$ & $42(95)$ \\
Mosaic attenuation & $22(82)$ & $11(65)$ & $33(75)$ \\
Emphysema & $2(7)$ & $0(0)$ & $2(5)$ \\
Interstitial fibrosis & $3(11)$ & $1(6)$ & $4(9)$ \\
Biopsy findings (n=42) & $22(81)$ & $12(75)$ & $34(77)$ \\
Carcinoid tumourlets & $12(44)$ & $9(53)$ & $21(50)$ \\
Constrictive bronchiolitis & $7(26)$ & $9(53)$ & $16(36)$ \\
Carcinoid tumour & $2(7)$ & $5(29)$ & $7(16)$ \\
Granuloma & $5(19)$ & $1(6)$ & $6(14)$ \\
Emphysema & $0(0)$ & $0(18)$ & $3(7)$ \\
Organising pneumonia & $1(4)$ & $0(0)$ & $1(2)$ \\
NSIP & $1(4)$ & $1(2)$ \\
Respiratory bronchiolitis & & & \\
\hline Data are presented as n (\%). NSIP: nonspecific interstitial pneumonitis. & \\
\hline
\end{tabular}


Octreotide, a somatostatin analogue (SSA), was prescribed in 14 patients (32\%); 4 (29\%) experienced symptomatic improvement, but only $1(13 \%)$ of 8 patients with available follow-up PFT data demonstrated an improvement on PFT. It is worth noting that $57 \%$ of the patients prescribed an SSA did not have a carcinoid tumour. Everolimus, in combination with cabozantinib and a programmed death ligand-1 inhibitor was prescribed in one patient with a metastatic pulmonary carcinoid tumour. This patient reported stable respiratory symptoms but manifested radiological progression of extrapulmonary metastases despite treatment.

One patient in our study underwent bilateral lung transplantation for progressive, multifactorial respiratory failure. This patient had surfactant protein C deficiency, with chronic bronchiolitis, bronchiolectasis, follicular bronchiolitis and interstitial fibrosis diagnosed by lung biopsy at 5 years of age. He underwent bilateral lung transplantation at the age of 21 and DIPNECH was diagnosed upon examination of the explanted lungs. The most recent PFT prior to transplantation had revealed a very severe mixed abnormality ( $\mathrm{FEV}_{1} 14 \%$ predicted) and air trapping (RV 272\%).

In the overall cohort, 35 patients (80\%) had at least one follow-up appointment at our institution, with a median follow-up interval of 30 months (IQR 14-52). Symptoms remained stable in $77 \%$ (27 out of 35), worsened in 3\% ( 1 out of 35) and improved in 20\% (7 out of 35) (table 5). Among the 24 patients (55\%) with available follow-up PFT data, 50\% remained stable, 33\% worsened and $17 \%$ improved over a median follow-up interval of 21.3 months (IQR 9.7-46.9) (table 6).

\section{Discussion}

DIPNECH is a rare disorder first described in 1992 [8]. According to the WHO's definition, DIPNECH diagnosis is solely made on pathology, irrespective of clinical, physiological or radiological parameters [15]. To the best of our knowledge, this study includes the largest single cohort of patients with DIPNECH evaluated for airway disease (i.e. "DIPNECH syndrome"). Among our patients, 61\% manifested airflow obstruction on PFTs and only a minority manifested symptomatic or objective response to bronchodilators, SSAs or other pharmacotherapies.

Similar to others, our cohort was predominantly comprised of middle-aged and older women, with a female to male ratio of $10: 1[10,11,13,19]$. The most common presenting symptoms were chronic cough and dyspnoea, with a median symptomatic duration of 10 years prior to DIPNECH diagnosis. Overall, $45 \%$ of our cohort, $50 \%$ and $63 \%$ of two other cohorts had been given a diagnosis of another obstructive lung disease prior to DIPNECH diagnosis $[10,19]$. Given its rarity, DIPNECH is under-recognised as a potential cause for obstructive lung disease, particularly in women and the diagnosis is often delayed.

On PFT, our cohort demonstrated obstruction with a frequency similar to that reported by NASSAR et al. [13] (52\% versus 54\%, respectively). Conversely, the cohort studied by CARR et al. [10] manifested obstruction markedly more than ours ( $87 \%$ versus $52 \%$, respectively). This notable difference might be related to: 1) all 30 patients reported by CARR et al. [10] manifested respiratory symptoms compared to

\section{TABLE 5 Characteristics of patients with clinical follow-up data}

$\begin{array}{lccc}\text { Symptom change } & \text { Improved } & \text { Worsened } & \text { Stable } \\ \text { Patients } & 7(20) & 1(3) & 27(77) \\ \text { History of an obstructive lung disease } & 4(57) & 1(100) & 11(41) \\ \text { PFT pattern } & 0(0) & 0(0) & 2(7) \\ \quad \text { Normal } & 4(57) & 1(100) & 15(56) \\ \text { Obstructive } & 1(14) & 0(0) & 3(11) \\ \text { Restrictive } & 0(0) & 0(0) & 1(4) \\ \text { Mixed } & 2(29) & 0(0) & 6(22) \\ \text { Nonspecific } & 1(14) & 1(100) & 7(26) \\ \text { Positive bronchodilator response } & 3(43) & 1(100) & 11(41) \\ \text { Constrictive bronchiolitis on biopsy } & & & 7(26) \\ \text { Treatment given } & 1(14) & 1(100) & 9(33) \\ \text { Observation } & 5(71) & 0(0) & 6(22) \\ \text { Inhaled } \beta_{2} \text {-agonist } & 2(29) & 0(0) & 10(37) \\ \text { Inhaled antimuscarinic } & 4(57) & 0(0) & 10(37) \\ \text { ICS } & 4(57) & & \end{array}$

Data are presented as $\mathrm{n}(\%)$. PFT: pulmonary function test; ICS: inhaled corticosteroid. 


\begin{tabular}{|c|c|c|c|}
\hline PFT change & Improved & Declined & Stable \\
\hline Patients & $4(17)$ & 8 (33) & $12(50)$ \\
\hline History of an obstructive lung disease & $2(50)$ & $3(38)$ & $5(42)$ \\
\hline \multicolumn{4}{|l|}{ PFT pattern } \\
\hline Normal & $0(0)$ & $1(13)$ & 1 (8) \\
\hline Obstructive & $2(50)$ & $5(63)$ & 7 (58) \\
\hline Restrictive & $1(25)$ & $1(13)$ & $2(17)$ \\
\hline Mixed & $0(0)$ & $1(13)$ & $1(8)$ \\
\hline Nonspecific & $1(25)$ & $0(0)$ & $1(8)$ \\
\hline Carcinoid tumour on biopsy & $2(50)$ & $5(63)$ & $7(58)$ \\
\hline \multicolumn{4}{|l|}{ Treatment given } \\
\hline Observation & $1(25)$ & $1(13)$ & 2 (17) \\
\hline Inhaled $\beta_{2}$-agonist & $3(75)$ & $4(50)$ & $7(58)$ \\
\hline Inhaled antimuscarinic & $2(50)$ & $4(50)$ & $6(50)$ \\
\hline ICS & $3(75)$ & $3(38)$ & $6(50)$ \\
\hline Octreotide & 1 (25) & 3 (38) & 4 (33) \\
\hline Mean $\mathrm{FEV}_{1} \%$ predicted initial/follow-up & $46 / 59$ & $55 / 50$ & $52 / 52$ \\
\hline
\end{tabular}

$86 \%$ in our cohort, and/or 2) the definition of obstruction used by CARR et al. [10] was not clearly stated and may have differed from the definition we used. Defining obstruction as $\mathrm{FEV}_{1} / \mathrm{FVC}<0.7$ rather than the definition we used can over-diagnose obstruction in older individuals [20].

The presumed aetiology of airflow obstruction in DIPNECH is narrowing of the bronchiolar lumen (i.e. $\mathrm{CB}$ ) owing to intra-luminal growth of PNECs, and/or mucosal and peribronchiolar fibrosis. It is surprising, however, that only $53 \%$ of our patients with airflow obstruction demonstrated $\mathrm{CB}$ on histological examination. Similarly, in a study of 30 patients with DIPNECH, $86 \%$ of whom manifested airflow obstruction, CB was observed in only 8 (44\%) of the 18 examined biopsies [10]. This finding can be explained by the patchy nature of $\mathrm{CB}$, which may have limited its identification upon histological examination [21]. However, the comparable frequency of $\mathrm{CB}$ among patients with and without airflow obstruction in our study ( $53 \%$ versus $44 \%$, respectively; $\mathrm{p}=0.58$ ), and the bronchodilator responsiveness, an unusual finding in $\mathrm{CB}$ [22], in some patients suggest that pathophysiological mechanisms other than $\mathrm{CB}$ contribute to airflow obstruction in DIPNECH.

Among patients who underwent bronchodilator responsiveness testing, $23 \%$ in this study, $33 \%$ and $10 \%$ in two other studies manifested a positive bronchodilator response [10,13]. This finding suggests that bronchospasm contributes to airflow obstruction in some patients with DIPNECH. Bronchospasm may simply be due to a comorbid reactive airway disease such as asthma, or, theoretically, may be related to increased amounts of bioactive substances (e.g. histamine and 5-hydroxytryptamine) secreted by the hyperplastic PNECs and carcinoid cells, and exerting their effect locally, on adjacent airways, rather than systemically $[23,24] ; 33$ patients in this study and 2 others had 5-HIAA measured; only one patient had 5-HIAA elevation $[10,19]$.

Although incompletely understood, tissue fibrosis (e.g. in mesenteric tissue and cardiac valves) is a well-known complication of neuroendocrine tumours (NETs). Serotonin and several growth factors have been implicated in its pathogenesis [25]. The most widely accepted pathophysiologic mechanism underlying the development of peribronchiolar fibrosis and $\mathrm{CB}$ in DIPNECH is that bioactive substances (e.g. bombesin) secreted in a larger-than-normal amount by the hyperplastic PNECs exert pro-fibrotic and pro-inflammatory effects on the adjacent airways [8, 26, 27]. This hypothesis triggered some physicians to propose two plausible therapeutic approaches to DIPNECH: 1) using SSAs to decrease the secretion of bioactive substances from the hyperplastic PNECs [10, 28]; and 2) using steroids (inhaled and/or systemic) to control inflammation and prevent further parenchymal and airway damage [13].

NASSAR et al. [13] summarised 25 cases of DIPNECH reported in the literature. Seven patients received systemic steroids; four improved ("clinically"), two remained stable and one worsened. One patient received ICS without systemic steroids and experienced respiratory decline. Overall, $46 \%$ and $67 \%$ of the patients studied by CARR et al. [10] received systemic and inhaled steroids, respectively. Although it is 
unclear how patients responded specifically to steroids, it is worth noting that $33 \%$ of their entire cohort continued to have respiratory decline. In our study, one patient received systemic steroids and remained stable. A total of 18 patients received ICSs; $25 \%$ experienced subjective improvement and 17\% manifested improvement on PFT.

The WHO considers DIPNECH as the precursor of other pulmonary NETs [15]. Studies have shown that pulmonary carcinoid tumours express somatostatin receptors (SSTRs) in a large percentage of patients [29], and the more differentiated a tumour is, the higher the likelihood of SSTR expression becomes [30]. SSTR expression in tumours can be detected noninvasively via different imaging modalities such as OctreoScan and the more sensitive, ${ }^{68}$ Gallium (Ga) DOTATATE scan [31, 32]. GorshteIn et al. [28] reported that OctreoScan was positive in 10 out of 11 patients with DIPNECH, but the uptake was exclusively found in the dominant lesion. Al-Toubah et al. [19] reported that some level of uptake on SSTR imaging (OctreoScan and ${ }^{68} \mathrm{Ga}$-DOTATATE) was noted in 12 of 19 patients with DIPNECH; however, it is unclear whether the uptake was exclusively in the dominant lesion as reported by GoRSHTEIN et al. [28] or more diffuse. The small size of the lesions found in DIPNECH negatively impacts the sensitivity of SSTR imaging modalities; hence, a negative study does not rule out the possibility of SSTR expression by those lesions $[33,34]$. Therefore, using SSAs in the treatment of DIPNECH, irrespective of the findings on SSTR imaging, is plausible.

SSA use has been reported in patients with DIPNECH [10, 19, 28, 35]. AL-ToubaH et al. [19] studied a cohort of 42 patients with DIPNECH, all of whom received an SSA; 11 patients (26\%) received an SSA alone, and $31(74 \%)$ received an SSA in combination with other therapies, including inhalers, steroids and benzonatate. Thirty-two patients (76\%) reported symptomatic improvement, and 14 of 15 patients with available pre- and post-treatment PFT data had an increase in $\mathrm{FEV}_{1}$, the magnitude of which was not specified. In another study, six patients with progressive DIPNECH received an SSA; four demonstrated improvement in $\mathrm{FEV}_{1}$, and two demonstrated stability [28]. In another study, 11 patients with DIPNECH received an SSA; 3 patients reported improvement of their cough, and all 9 follow-up PFTs demonstrated stability [10]. A small case series reported drastic improvement of cough in all four patients treated with SSA [35]. In a small case series, one patient with DIPNECH received an SSA, with temporary improvement in her cough [36]. In our study, 14 patients received an SSA; 4 (29\%) experienced improvement in their cough. Among the eight patients with follow-up PFTs, one (12.5\%) improved, three (37.5\%) worsened and four (50\%) remained stable.

Some NETs demonstrate aberrant activation of the mechanistic target of rapamycin (mTOR) pathway, which led to the successful use of everolimus, an mTOR inhibitor, in improving outcomes in patients with NETs of different origins [37]. This activation of mTOR pathway was also demonstrated in DIPNECH [38]. This led to the use of sirolimus, another mTOR inhibitor, in the management of three patients with progressive DIPNECH and presumed carcinoid tumours. Two patients had radiological improvement, one patient had radiological progression, and all three patients demonstrated improvement in $\mathrm{FEV}_{1}$ over time [39]. In our study, one patient received everolimus for metastatic pulmonary carcinoid. This patient reported stable symptoms, but manifested radiological progression on follow-up.

In a study of 55 patients with resected carcinoid tumours, 3 patients (5\%) were found to have DIPNECH on histopathology. Those 3 patients did not behave differently than the 52 patients without DIPNECH during a 37-month follow-up. Although the sample size is too small and no statistical testing was feasible, the authors concluded that coexisting DIPNECH is probably of no clinical consequence following the resection of the carcinoid tumour [40]. In the study by AL-Touba et al. [19], 37 of 42 patients (88\%) underwent surgical resection of at least one tumour. However, the outcomes for the group that underwent resection were not reported separately. In another study, 9 of 11 patients with DIPNECH and carcinoid tumour underwent surgical resection of the dominant lesion. In this study, 5 of 11 patients remained stable, while 6 progressed requiring treatment with SSA [28]. In our study, 5 of 12 patients (42\%) with follow-up data, reported improved respiratory symptoms following resection of the dominant carcinoid lesion.

Similar to others, our study demonstrates that DIPNECH remains stable or progresses very slowly in most patients, but can result in severe airflow limitation and respiratory impairment $[8,10,13,26,28,35,36]$.

Our study has limitations inherent to the retrospective single-centre design and the rarity of the disease under study. The number of subjects in this study is modest and the features described within may reflect the more severe end of the disease spectrum due to referral bias. Some data were missing, and several patients were lost to follow-up at our institution.

\section{Conclusion}

DIPNECH syndrome is probably an under-recognised form of obstructive lung disease in middle-aged and older women, often misdiagnosed as asthma or COPD. DIPNECH typically manifests diffuse pulmonary 
nodules on chest CT scan, often associated with mosaic attenuation. DIPNECH syndrome can progress slowly and sometimes lead to respiratory failure. Response to currently employed therapies seems limited and optimal management strategies need to be identified, ideally in the context of well-designed prospective trials.

Conflict of interest: B.F. Samhouri has nothing to disclose. N. Azadeh has nothing to disclose. T.R. Halfdanarson reports advisory boards (money paid to institution) for Advanced Accelerator Applications and Ipsen, personal fees for advisory boards from Curium and ScioScientific, and research support from Ipsen, outside the submitted work. E.S. Yi has nothing to disclose. J.H. Ryu has nothing to disclose.

\section{References}

1 Boers JE, Den Brok JLM, Koudstaal J, et al. Number and proliferation of neuroendocrine cells in normal human airway epithelium. Am J Respir Crit Care Med 1996; 154: 758-763.

2 Gosney JR, Sissons MCJ, Allibone RO. Neuroendocrine cell populations in normal human lungs: a quantitative study. Thorax 1988; 43: 878-882.

3 Kemp PJ, Lewis A, Hartness ME, et al. Airway chemotransduction: from oxygen sensor to cellular effector. Am J Respir Crit Care Med 2002; 166: S17-S24.

4 Branchfield K, Nantie L, Verheyden JM, et al. Pulmonary neuroendocrine cells function as airway sensors to control lung immune response. Science 2016; 351: 707-710.

5 Yousem SA, Isaacs A. Pulmonary neuroendocrine cells in the airways of lung allografts. Transplantation 1995; 59: 1070-1073.

6 Gould VE, Linnoila RI, Memoli VA, et al. Neuroendocrine components of the bronchopulmonary tract: hyperplasias, dysplasias, and neoplasms. Lab Invest 1983; 49: 519-537.

7 Gosney JR, Sissons MCJ, Allibone RO, et al. Pulmonary endocrine cells in chronic bronchitis and emphysema J Pathol 1989; 157: 127-133.

8 Aguayo SM, Miller YE, Waldron JA, et al. Idiopathic diffuse hyperplasia of pulmonary neuroendocrine cells and airways disease. N Engl J Med 1992; 327: 1285-1288.

9 Brambilla E, Travis WD, Colby T V, et al. The new World Health Organization classification of lung tumours. Eur Respir J 2001; 18: 1059-1068.

10 Carr LL, Chung JH, Achcar RD, et al. The clinical course of diffuse idiopathic pulmonary neuroendocrine cell hyperplasia. Chest 2015; 147: 415-422.

11 Marchevsky AM, Wirtschafter E, Walts AE. The spectrum of changes in adults with multifocal pulmonary neuroendocrine proliferations: what is the minimum set of pathologic criteria to diagnose DIPNECH? Hum Pathol 2015; 46: 176-181.

12 Hansell DM, Bankier AA, MacMahon H, et al. Fleischner Society: glossary of terms for thoracic imaging Radiology 2008; 246: 697-722.

13 Nassar AA, Jaroszewski DE, Helmers RA, et al. Diffuse idiopathic pulmonary neuroendocrine cell hyperplasia: a systematic overview. Am J Respir Crit Care Med 2011; 184: 8-16.

14 Rossi G, Cavazza A, Spagnolo P, et al. Diffuse idiopathic pulmonary neuroendocrine cell hyperplasia syndrome. Eur Respir J 2016; 47: 1829-1841.

15 Travis WD, Brambilla E, Nicholson AG, et al. The 2015 World Health Organization classification of lung tumors: impact of genetic, clinical and radiologic advances since the 2004 classification. J Thorac Oncol 2015; 10: $1243-1260$.

16 Pellegrino R, Viegi G, Brusasco V, et al. Interpretative strategies for lung function tests. Eur Respir J 2005; 26 948-968.

17 Iyer VN, Schroeder DR, Parker KO, et al. The nonspecific pulmonary function test: longitudinal follow-up and outcomes. Chest 2011; 139: 878-886.

18 Aggarwal AN, Agarwal R. The new ATS/ERS guidelines for assessing the spirometric severity of restrictive lung disease differ from previous standards. Respirology 2007; 12: 759-762.

19 Al-Toubah T, Strosberg J, Halfdanarson TR, et al. Somatostatin analogs improve respiratory symptoms in patients with diffuse idiopathic neuroendocrine cell hyperplasia. Chest 2020; 158: 401-405.

20 Meteran $\mathrm{H}$, Miller MR, Thomsen SF, et al. The impact of different spirometric definitions on the prevalence of airway obstruction and their association with respiratory symptoms. ERJ Open Res 2017; 3: 00110-02017.

21 Silva CIS, Müller NL. Obliterative Bronchiolitis. In: CT Airways. Totowa, NJ, Humana Press, 2008; pp. $293-323$.

22 Krishna R, Anjum F, Oliver TI. Bronchiolitis Obliterans (Obliterative Bronchiolitis, Constrictive Bronchiolitis). In: StatPearls. Treasure Island, StatPearls Publishing, 2020.

23 Soga J, Yakuwa Y, Osaka M. Carcinoid syndrome: a statistical evaluation of 748 reported cases. J Exp Clin Cancer Res 1999; 18: 133-141.

24 Ferrari AC, Glasberg JP, Riechelmann RP. Carcinoid syndrome: update on the pathophysiology and treatment Clinics (Sao Paulo) 2018; 73: e490s.

25 Laskaratos FM, Rombouts K, Caplin M, et al. Neuroendocrine tumors and fibrosis: an unsolved mystery? Cancer 2017: 4770-4790.

26 Degan S, Lopez GY, Kevill K, et al. Gastrin-releasing peptide, immune responses, and lung disease. Ann NY Acad Sci 2008; 1144: 136-147.

27 Zhou S, Nissao E, Jackson IL, et al. Radiation-induced lung injury is mitigated by blockade of gastrin-releasing peptide. Am J Pathol 2013; 182: 1248-1254.

28 Gorshtein A, Gross DJ, Barak D, et al. Diffuse idiopathic pulmonary neuroendocrine cell hyperplasia and the associated lung neuroendocrine tumors. Cancer 2012; 118: 612-619.

29 Reubi JC, Waser B, Kvols LK, et al. Detection of somatostatin receptors in surgical and percutaneous needle biopsy samples of carcinoids and islet cell carcinomas. Cancer Res 1990; 50: 5969-5977. 
30 Righi L, Volante M, Tavaglione V, et al. Somatostatin receptor tissue distribution in lung neuroendocrine tumours: a clinicopathologic and immunohistochemical study of 218 "clinically aggressive" cases. Ann Oncol 2010; 21 548-555.

31 Deppen SA, Blume J, Bobbey AJ, et al. 68Ga-DOTATATE compared with 111In-DTPA-octreotide and conventional imaging for pulmonary and gastroenteropancreatic neuroendocrine tumors: a systematic review and meta-analysis. J Nucl Med 2016; 57: 872-878.

32 Hope TA, Calais J, Zhang L, et al. 111In-pentetreotide scintigraphy versus 68Ga-DOTATATE PET: impact on krenning scores and effect of tumor burden. J Nucl Med 2019; 60: 1266-1269.

33 Maxwell JE, Howe JR. Imaging in neuroendocrine tumors: an update for the clinician. Int J Endocr Oncol 2015; 2: $159-168$.

34 Maxwell JE, Sherman SK, Menda Y, et al. Limitations of somatostatin scintigraphy in primary small bowel neuroendocrine tumors. J Surg Res 2014; 190: 548-553.

35 Chauhan A, Ramirez RA. Diffuse idiopathic pulmonary neuroendocrine cell hyperplasia (DIPNECH) and the role of somatostatin analogs: a case series. Lung 2015; 193: 653-657.

36 Cabezón-Gutiérrez L, Khosravi-Shahi P, Palka-Kotlowska M, et al. Diffuse idiopathic pulmonary neuroendocrine cell hyperplasia: review of the literature and a single-center experience. Cureus 2019; 11: e5640.

37 Yao JC, Fazio N, Singh S, et al. Everolimus for the treatment of advanced, non-functional neuroendocrine tumours of the lung or gastrointestinal tract (RADIANT-4): a randomised, placebo-controlled, phase 3 study. Lancet 2016; 387: 968-977.

38 Rossi G, Cavazza A, Graziano P, et al. mTOR/p70S6K in diffuse idiopathic pulmonary neuroendocrine cell hyperplasia. Am J Respir Crit Care Med 2012; 185: 341.

39 Russier M, Plantier L, Derot G, et al. Diffuse idiopathic pulmonary neuroendocrine cell hyperplasia syndrome treated with sirolimus. Ann Intern Med 2018; 169: 197-198.

40 Ruffini E, Bongiovanni M, Cavallo A, et al. The significance of associated pre-invasive lesions in patients resected for primary lung neoplasms1. Eur J Cardio-Thoracic Surg 2004; 26: 165-172. 